\author{
7-9 MARCH ,2019 London,United Kingdom
}

\title{
An Analysis of the Effect of Emotional Eating on both Eating Behaviours and Obesity among Adolescents in KSA
}

\author{
Maali Aloudah \\ University of Reading
}

\begin{abstract}
Emotional eating is an eating behaviour, when someone consuming food in responding to an emotional motivation rather than to a real hunger. These emotional motivations include happiness, sadness, anxiety, depression, fear, anger and loneliness. This could be attributed to psychological, social and cultural factors. Emotional eating is usually identified by over eating food that contains a large amount of energy and fat. It has well been proved that there is a positive relationship between emotional eating and both obesity and disordered eating. It is known that obesity and disordered eating are highly widespread among adolescents in the world. On the other hand, literature considers emotional eating as one of the main factors that causes eating disorders among people. It is important to determine the correlation between eating food and feelings because this information helps in reducing the overeating problem. It is known that both eating disorders and obesity have negative impacts on emotional well-being in the long term, which is difficult to be treated or overcome through traditional way. Therefore, raising awareness about emotional eating and encouraging healthy eating could play a key role in reducing the emotional eating and unhealthy eating behaviours among adolescents. This study uses a mixedmethod through collecting data using questionnaire and oneto-one interview tools. A number of 300 students (ages 12-15) from Riyadh (the capital city of Saudi Arabia) have completed a questionnaire, which designed for this purpose. On the other hand, a number of 15 female students have engaged in a one-to-one interview. The results show that $81.5 \%$ of students have emotional eating, however this varies according to the level of the emotional eating among the participants as $27 \%, 34 \%$ and $20.3 \%$ of them reported to have high, moderate and weak levels of emotional eating, respectively. The study finds that the factors that promote emotional eating are as follows: Feeling, parents as well as peers and subsequently, social media. Additionally, the majority of the participants (70\% of the boys and $94.73 \%$ of the girls) are indulged positively in emotional eating (happy eating). The study reports the main factors that encourage boys and girls are as follows, respectively: eating food for the sense of comfort (39.3\% and $72 \%$ ), anxiety ( $28 \%$ and $54 \%)$, sadness (17.3\% and $34 \%)$, and to feel safe (32\% and $36 \%)$, while hunger is $(11.3 \%$ and $6 \%)$.
\end{abstract}

Keywords: eating; eating disorders; emotions; students; Saudi Arabia. 